\title{
Fibroblasts Induce Calcium Alternans When Coupled to Cardiomyocytes: A Simulation Study
}

\author{
Maria T Mora, Albert Dasi, Javier Saiz, Beatriz Trenor, Jose M Ferrero \\ Universitat Politècnica de València, Valencia, Spain
}

\begin{abstract}
Cardiac alternans has been linked to arrhythmias, and certain conditions, such as fibrosis, can change the onset of alternans increasing the vulnerability to electrical instabilities. The study of the underlying mechanisms of beat-to-beat cellular fluctuations and, in particular, when fibroblasts interact with myocytes, could help to reduce the risk of reentrant activity.

We investigated the origin of cellular alternans in myocytes coupled to fibroblasts using mathematical models. Differences in alternans development in relation to variability in ion transport pathways between individuals of a population of cells was the key to identify the parameters that can modulate cellular alternation.

Repolarization alternans and $\mathrm{Ca}^{2+}$ cycling alternans were concomitant in myocytes, but the latter was a better quantitative indicator due to marked alternating $\mathrm{Ca}^{2+}$ transients. Despite the similar mechanisms inducing $\mathrm{Ca}^{2+}$ alternans, the myocyte-fibroblast population was more prone to alternans than the population without fibroblast. Our findings suggest that $\mathrm{Ca}^{2+}$-related parameters regulate alternans formation because they occur due to an impaired $\mathrm{Ca}^{2+}$ dynamics, a situation that is exacerbated by fibrosis.
\end{abstract}

\section{Introduction}

Myocardial remodeling in heart diseases and aging involves a damaging proliferation of fibroblasts in cardiac tissue, affecting the mechanical and electrical function of the myocardium. Fibrosis has been linked to an increased risk of reentrant arrhythmias, generally by slowing impulse propagation and causing conduction block [1]. However, an electrical active role of fibroblasts could be causing electrical instabilities in myocytes, increasing the vulnerability to arrhythmogenesis by means of alternans development.

Electrotonic interactions between myocytes and fibroblasts, coupled by gap junctional-type connections, modulate the electrophysiology of cardiomyocytes. The resultant reduced action potential (AP) duration (APD) and depressed intracellular $\mathrm{Ca}^{2+}$ transient (CaT) indicate that there are changes in sarcolemmal currents and $\mathrm{Ca}^{2+}$ transport mechanisms, which could alter cellular alternans formation. The origin of alternans has been the center of many investigations due to the associated risk of sudden cardiac death [2-4], but the role of fibrosis has not been widely investigated. Therefore, we focused on the effects of myocyte-fibroblast interactions on cellular alternans.

To better understand the cellular origin of alternans, the study of ionic transport mechanisms regulating both the electrical and mechanical activity at the cellular level is crucial, due to the bidirectional coupling between membrane voltage and $\mathrm{Ca}^{2+}$ cycling. The use of mathematical AP models facilitates the exploration of the multiple ionic mechanisms and complex interactions to explain experimental findings. Biological variability causes different electrophysiological responses, and the study of cell-to-cell differences in maximal conductances and fluxes of ionic transport processes could reveal mechanisms promoting alternans formation.

In this study, we used modeling and computer simulations to gain insight into the mechanisms of alternans formation. In particular, we assessed the active role of fibroblasts in modulating myocyte's susceptibility to beat-to-beat alternation. The ionic transport processes acting in the development of alternans, obtained by analyzing inter-subject variability, are the key pharmacological targets to avoid arrhythmogenic electrical fluctuations.

\section{Methods}

In order to evaluate the onset of alternans in myocytes at fast pacing rates and the potential role of coupled fibroblasts in this phenomenon, we conducted simulations in which a single myocyte was either isolated or coupled to a certain number of fibroblasts. The human AP model by O'Hara et al. [5] was used to simulate the electrophysiological activity of endocardial myocytes and, when considering fibrosis, the active fibroblast model by MacCannell et al. [6] was coupled to a myocyte. Myocytefibroblast coupling was performed using an individual myocyte-fibroblast coupling conductance of $\mathrm{G}_{\text {gap }}$ of $3 \mathrm{nS}$, 
within the experimental range [7], which was increased five-fold to establish a 1:5 ratio between cells, a surrogate for an increased degree of fibrosis.

The restitution curve of myocytes and myocytefibroblast aggregations was computed by reducing pacing cycle length (CL) from 1000 ms until loss of capture of AP. We recorded the last two AP and measured AP duration from maximal upstroke to $90 \%$ of repolarization $\left(\mathrm{APD}_{90}\right)$ and the systolic peak of intracellular $\mathrm{Ca}^{2+}$.

To generate results representing natural variability, a population of models was generated by varying the maximum values of 13 parameters of the myocyte, accounting for maximal ionic conductances and fluxes [8]. The selected variables were the fast $\mathrm{Na}^{+}$current $\left(\mathrm{I}_{\mathrm{Na}}\right)$, the late $\mathrm{Na}^{+}$current $\left(\mathrm{I}_{\mathrm{NaL}}\right)$, the transient outward $\mathrm{K}^{+}$current $\left(\mathrm{I}_{\mathrm{to}}\right)$, the $\mathrm{L}$-type $\mathrm{Ca}^{2+}$ current $\left(\mathrm{I}_{\mathrm{CaL}}\right)$, the rapid delayed rectifier $\mathrm{K}^{+}$current $\left(\mathrm{I}_{\mathrm{Kr}}\right)$, the slow delayed rectifier $\mathrm{K}^{+}$ current $\left(\mathrm{I}_{\mathrm{Ks}}\right)$, the inward rectifier $\mathrm{K}^{+}$current $\left(\mathrm{I}_{\mathrm{K} 1}\right)$, the $\mathrm{Na}^{+} /$ $\mathrm{K}^{+}$ATPase current $\left(\mathrm{I}_{\mathrm{NaK}}\right)$, the $\mathrm{Na}^{+} / \mathrm{Ca}^{2+}$ exchange current ( $\mathrm{I}_{\mathrm{NCX}}$ ), the sarcoplasmic reticulum (SR) $\mathrm{Ca}^{2+}$ uptake via SERCA pump (J JERCA), the SR Ca ${ }^{2+}$ release flux via RyR $\left(\mathrm{J}_{\text {rel }}\right)$, the SR $\mathrm{Ca}^{2+}$ leak $\left(\mathrm{J}_{\text {leak }}\right)$, and the $\mathrm{Na}^{+}$background current $\left(\mathrm{I}_{\mathrm{Nab}}\right)$. Parameters were modified applying random scale factors obtained from a normal distribution (mean=1, $\mathrm{SD}=0.15)$. Two populations of 300 different human AP models, one for isolated myocytes and another for myocyte-fibroblast coupling, were obtained and paced at a fixed CL of $300 \mathrm{~ms}$. The study of ionic mechanisms that facilitate beat-to-beat oscillations was performed taking into account this cell-to-cell variability. Alternans was calculated as fluctuations in $\mathrm{CaT}$ amplitude between two consecutive beats, defined as

$$
\text { Ca_alt }=1-A_{\text {smallcat }} / A_{\text {largecaT }},
$$

where A represents the amplitude of the small or large $\mathrm{CaT}$, respectively. Only models with $\mathrm{Ca}$-alt $>0.1$ were classified in the alternans group. APD alternans (APD-alt) was also determined, as follows:

$$
A P D \_a l t=A P D 90_{\text {largecaT }}-A P D 90_{\text {smallcat }}
$$

Statistical differences in parameters between groups were determined with the Wilcoxon rank-sum test.

\section{Results and Discussion}

Beat-to-beat alternation of APD and CaT amplitude, which occurs simultaneously, arises at fast pacing rates ( $C L<400 \mathrm{~ms}$ ), and their magnitude increases as CL is reduced until loss of capture of AP (Figure 1). The basic myocyte-fibroblasts model presents an earlier onset of alternans compared to the single myocyte model (370 vs $310 \mathrm{~ms}$ ), indicating that fibrosis promotes alternans. Although the variation of $\mathrm{APD}_{90}$ between two consecutive beats seems small, it may induce spatial discordance in cardiac tissue, generate reentrant activity and finally lead to ventricular fibrillation $[2,9]$.

The electrotonic effects of fibroblasts on myocytes could be modulating the electrical activity of cells in a manner that induces alternans. The main differences between a single myocyte and a myocyte coupled to fibroblasts are a reduction in APD and a decrease in intracellular $\mathrm{Ca}^{2+}$ concentration (results not shown). When APD is shortened, the APD restitution curve becomes flatter, which can be observed in Figure 1. Thus, the theory that APD-alternans is due to a steep slope [10] does not apply in this case, suggesting another origin for alternans. Previous investigations have also determined that alternans are driven by $\mathrm{Ca}^{2+}$ oscillations due to intracellular $\mathrm{Ca}^{2+}$ instabilities [4,11,12], which would better explain why fibroblasts promote alternans when coupled to myocytes.
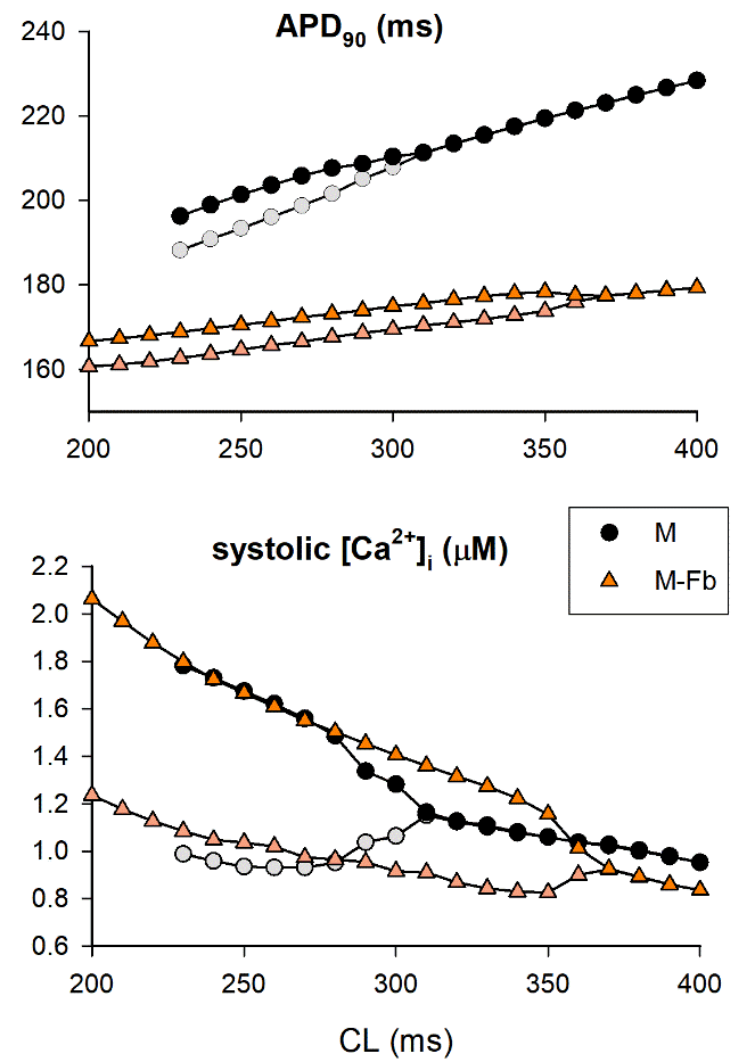

Figure 1. Restitution curve. APD ${ }_{90}$ and systolic $\left[\mathrm{Ca}^{2+}\right]_{\mathrm{i}}$ for two consecutive beats (dark and light symbols) in an isolated myocyte (M) and a myocyte coupled to 5 fibroblasts (M-Fb), using baseline models.

To further understand the origin of alternans with fibrosis, we compared ionic mechanisms in populations of myocytes coupled or not to fibroblasts. The selection of alternans cases was made considering the occurrence of Ca-alt because, unlike beat-to-beat variation of APD, CaT oscillations are more noticeable, and Ca-alt becomes a better quantitative indicator. 
Results from the populations, with pacing fixed at CL = $300 \mathrm{~ms}$, are consistent with the basic models, confirming that fibroblasts facilitate alternans generation: while 33\% of isolated myocytes present cellular alternans, this value increases to $47 \%$ in coupled myocyte-fibroblast model. Figure 2 illustrates the relationship between APD-alt and Ca-alt of all the cardiac cell models, showing a linear correlation between variables and the existence of electromechanical concordance, i.e. a large CaT is always associated with a long APD and vice versa.

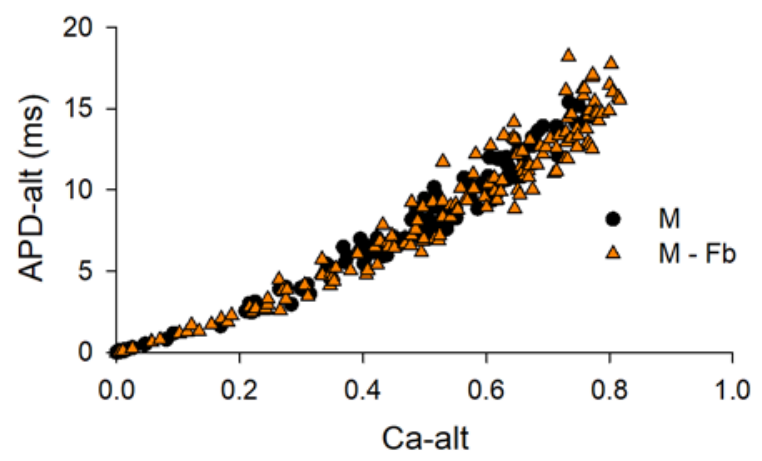

Figure 2. Intracellular $\mathrm{Ca}^{2+}$ alternans (Ca-alt) and $\mathrm{APD}_{90}$ alternans (APD-alt) relationship in populations of models. Single myocyte (M) and myocyte-fibroblast coupling (M$\mathrm{Fb})$.

In Figure 3, scale factors for each parameter that lead to alternans are compared with those that do not, showing some significant differences. In myocytes without coupled fibroblasts, alternans occur with higher values of $\mathrm{I}_{\mathrm{NCX}}$ and $\mathrm{J}_{\text {rel, }}$ and lower $\mathrm{J}_{\text {SERCA }}$ values, which is in agreement with the results obtained by Zhou et al [13]. These mechanisms are linked to $\mathrm{Ca}^{2+}$ cycling, as has been previously reported in the study of alternans [4,11,12]. Other significant differences between both groups are $\mathrm{I}_{\mathrm{Na}}, \mathrm{I}_{\mathrm{NaK}}$ and $\mathrm{I}_{\mathrm{Nab}}$. In the classification of myocyte-fibroblast models, we observe the same ionic mechanisms related to $\mathrm{Ca}^{2+}$ dynamics involved in alternans formation. Furthermore, the reduction of $\mathrm{I}_{\mathrm{CaL}}$ becomes important.

One theory for $\mathrm{Ca}^{2+}$ instabilities inducing alternans is the steep slope of the $\mathrm{SR} \mathrm{Ca}^{2+}$ load - $\mathrm{Ca}^{2+}$ release curve [14]. The amount of $\mathrm{Ca}^{2+}$ released through RyR channels depends on the SR $\mathrm{Ca}^{2+}$ content before the release occurs. At high frequencies, CaT must be larger and shorter, requiring a rapid $\mathrm{Ca}^{2+}$ release and posterior removal. A minor imbalance between mechanisms could be enough to initiate oscillations in the diastolic SR $\mathrm{Ca}^{2+}$ load [3], due to an incomplete process in one beat, followed by an exacerbated release in the following beat.
All $\mathrm{Ca}^{2+}$-handling proteins contribute to maintain normal $\mathrm{Ca}^{2+}$ cycling in myocytes to assure contractility, and a disruption of these proteins leads to abnormal $\mathrm{Ca}^{2+}$ homeostasis in all cell compartments. Because of an unbalanced $\mathrm{Ca}^{2+}$ dynamics, a loss of intracellular $\mathrm{Ca}^{2+}$ may also occur, with a reduction of SR $\mathrm{Ca}^{2+}$ load. This was observed in myocytes coupled to fibroblasts (results not shown) and the resulting impairment could facilitate the induction of alternans.

An enhanced NCX and $\mathrm{J}_{\mathrm{rel}}$, and an inhibition of SERCA and $\mathrm{I}_{\mathrm{CaL}}$, mechanisms that induce more alternans, all contribute to depress intracellular $\mathrm{Ca}^{2+}$ directly or indirectly by reducing SR $\mathrm{Ca}^{2+}$ content. It is known that SERCA downregulation leads to a reduced and slow CaT. Approaches to recover normal $\mathrm{Ca}^{2+}$ cycling include SERCA overexpression, and these have also proved to yield good results in delaying the development of alternans in intact hearts of guinea pigs and in reducing ventricular arrhythmias [15]. The refractoriness of RyR channels is not present in the ORd model and the higher development of alternans increasing $\mathrm{J}_{\text {rel }}$ occurs due to $\mathrm{Ca}^{2+}$ instabilities.

Finally, intracellular $\mathrm{Ca}^{2+}$ fluctuations are translated to repolarization alternans by alternating APD through electrogenic $\mathrm{Ca}^{2+}$ mechanisms, i.e. NCX and $\mathrm{I}_{\mathrm{CaL}}$ [16]. As Ca-V coupling was positive, NCX forward mode predominates, thus lengthening APD. The role of $\mathrm{I}_{\mathrm{CaL}}$ is minor, and its decrease leading to alternans in the presence of fibrosis highlights NCX as the main electrogenic mechanism governing $\mathrm{Ca}-\mathrm{V}$ coupling.

\section{Conclusions}

$\mathrm{Ca}^{2+}$-related mechanisms, such as NCX, SERCA, I $\mathrm{IaL}_{\mathrm{L}}$ and $\mathrm{J}_{\text {rel }}$ can induce alternans when $\mathrm{Ca}^{2+}$ dynamics is depressed, suggesting that this is the cellular origin of alternans. The greater impairment of $\mathrm{Ca}^{2+}$ cycling in myocytes coupled to fibroblasts would explain the higher vulnerability to alternans under these conditions and a correct modulation of ionic transport mechanisms could prevent arrhythmogenic cardiac alternans.

\section{Acknowledgements}

This work was partially supported by the "Plan Estatal de Investigación Científica y Técnica y de Innovación 2013-2016” from the Ministerio de Economía, Industria y Competitividad of Spain and Fondo Europeo de Desarrollo Regional (FEDER) DPI2016-75799-R (AEI/FEDER, UE), and by "Programa de Ayudas de Investigación y Desarrollo (PAID-01-17)” from the Universitat Politècnica de València. 


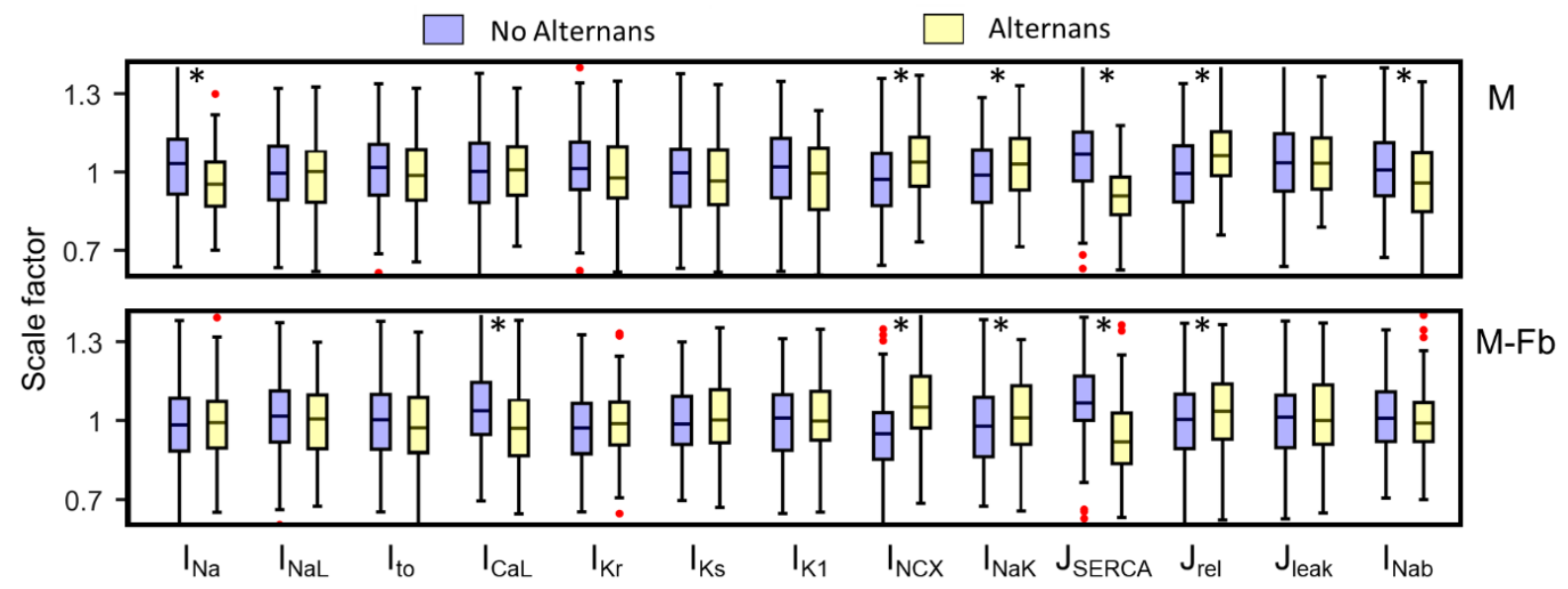

Figure 3. Comparison of parameter distribution for alternans and non-alternating models $\left({ }^{*} \mathrm{p}<0.05\right)$ in a population of single myocytes (M) and a myocyte-fibroblast population (M-Fb).

\section{References}

[1] S. Zlochiver, V. Muñoz, K. L. Vikstrom, S. M. Taffet, O. Berenfeld, and J. Jalife, "Electrotonic myofibroblast-tomyocyte coupling increases propensity to reentrant arrhythmias in two-dimensional cardiac monolayers.," Biophys. J., vol. 95, no. 9, pp. 4469-80, Nov. 2008.

[2] L. Wang, R. C. Myles, N. M. De Jesus, A. K. P. Ohlendorf, D. M. Bers, and C. M. Ripplinger, "Optical mapping of sarcoplasmic reticulum $\mathrm{Ca} 2+$ in the intact heart: Ryanodine receptor refractoriness during alternans and fibrillation,” Circ. Res., vol. 114, no. 9, pp. 1410-1421, 2014.

[3] M. E. Díaz, S. C. O’Neill, and D. A. Eisner, “Sarcoplasmic Reticulum Calcium Content Fluctuation Is the Key to Cardiac Alternans,” Circ. Res., vol. 94, no. 5, pp. 650-656, 2004.

[4] E. Picht, J. DeSantiago, L. A. Blatter, and D. M. Bers, "Cardiac alternans do not rely on diastolic sarcoplasmic reticulum calcium content fluctuations,” Circ. Res., vol. 99, no. 7, pp. 740-748, 2006.

[5] T. O’Hara, L. Virág, A. Varró, and Y. Rudy, "Simulation of the undiseased human cardiac ventricular action potential: model formulation and experimental validation.," PLoS Comput. Biol., vol. 7, no. 5, p. e1002061, 2011.

[6] K. A. MacCannell, H. Bazzazi, L. Chilton, Y. Shibukawa, R. B. Clark, and W. R. Giles, "A mathematical model of electrotonic interactions between ventricular myocytes and fibroblasts.,” Biophys. J., vol. 92, no. 11, pp. 4121-4132, 2007.

[7] M. B. Rook, A. C. van Ginneken, B. de Jonge, A. el Aoumari, D. Gros, and H. J. Jongsma, "Differences in gap junction channels between cardiac myocytes, fibroblasts, and heterologous pairs,” Am. J. Physiol. Physiol., vol. 263, no. 5, pp. C959-C977, Nov. 1992.

[8] O. J. Britton et al., "Experimentally calibrated population of models predicts and explains intersubject variability in cardiac cellular electrophysiology,” Proc. Natl. Acad. Sci., vol. 110, no. 23, pp. E2098-E2105, Jun. 2013.

[9] R. Majumder, M. C. Engels, A. A. F. de Vries, A. V.
Panfilov, and D. A. Pijnappels, "Islands of spatially discordant APD alternans underlie arrhythmogenesis by promoting electrotonic dyssynchrony in models of fibrotic rat ventricular myocardium," Sci. Rep., vol. 6, no. 1, p. 24334, Jul. 2016.

[10] J. B. Nolasco and R. W. Dahlen, "A graphic method for the study of alternation in cardiac action potentials.,” J. Appl. Physiol., vol. 25, no. 2, pp. 191-196, 1968.

[11] E. J. Pruvot, R. P. Katra, D. S. Rosenbaum, and K. R. Laurita, "Role of calcium cycling versus restitution in the mechanism of repolarization alternans.,” Circ. Res., vol. 94, no. 8, pp. 1083-90, Apr. 2004.

[12] L. D. Wilson, X. Wan, and D. S. Rosenbaum, “Cellular alternans: A mechanism linking calcium cycling proteins to cardiac arrhythmogenesis,” Ann. N. Y. Acad. Sci., vol. 1080, pp. 216-234, 2006.

[13] X. Zhou et al., "In vivo and in silico investigation into mechanisms of frequency dependence of repolarization alternans in human ventricular cardiomyocytes,” Circ. Res., vol. 118, no. 2, pp. 266-278, Jan. 2016.

[14] D. A. Eisner, H. S. Choi, M. E. Diaz, S. C. O’Neill, and A. W. Trafford, "Integrative Analysis of Calcium Cycling in Cardiac Muscle,” Circ. Res., vol. 87, no. 12, pp. 1087-1094, Dec. 2000.

[15] M. J. Cutler, X. Wan, K. R. Laurita, R. J. Hajjar, and D. S. Rosenbaum, “Targeted SERCA2a Gene Expression Identifies Molecular Mechanism and Therapeutic Target for Arrhythmogenic Cardiac Alternans," Circ. Arrhythmia Electrophysiol., vol. 2, no. 6, pp. 686-694, Dec. 2009.

[16] X. Wan et al., "New experimental evidence for mechanism of arrhythmogenic membrane potential alternans based on balance of electrogenic INCX/ICa currents,” Hear. Rhythm, vol. 9, no. 10, pp. 1698-1705, Oct. 2012.

Address for correspondence:

Maria Teresa Mora

Ci2B, Universitat Politècnica de València

Camino de Vera s/n, 46022 Valencia, Spain

mamofe@doctor.upv.es 